\title{
Formation of Ellerman bombs due to 3D flux emergence
}

\author{
V. Archontis and A. W. Hood
}

\author{
School of Mathematics and Statistics, University of St. Andrews, North Haugh, St. Andrews, Fife, KY16 9SS, UK \\ e-mail: [vasilis;alan]@mcs.st-and.ac.uk
}

Received 8 May 2009 / Accepted 21 October 2009

\section{ABSTRACT}

\begin{abstract}
Aims. We investigate the emergence of a "sea-serpent" magnetic field into the outer solar atmosphere and the connection between undulating fieldlines and formation of Ellerman bombs.

Methods. We perform 3D numerical experiments solving the time-dependent and resistive MHD equations.

Results. A sub-photospheric magnetic flux sheet develops undulations due to the Parker instability. It rises from the convectively unstable sub-photospheric layer and emerges into the highly stratified atmosphere through successive reconnection events along the undulating system. Brightenings with the characteristics of Ellerman bombs are produced due to reconnection, which occurs during the emergence of the field. At an advanced stage of the evolution of the system, the resistive emergence leads to the formation of long, arch-like magnetic fields that expand into the corona. The enhancement of the magnetic field at the low atmosphere and episodes of emergence of new magnetic flux are also discussed.
\end{abstract}

Key words. Sun: magnetic fields - Sun: corona - magnetohydrodynamics (MHD) - methods: numerical - Sun: activity

\section{Introduction}

The emergence of magnetic flux from the solar interior leads to the appearance of emerging flux regions (EFRs) when the rising field intersects the visible surface of the Sun. A typical model for flux emergence relies on the buoyant rise of a sub-photospheric flux tube, which adopts an $\Omega$-like shape through the process of emergence. A classical bipolar active region may form as a result of the intersection of the $\Omega$-loop with the photosphere (Zwaan 1985). EFRs may also appear as growing arch-filament systems in $\mathrm{H}_{\alpha}$ or as a system of coronal loops recorded in UV when the emerging fieldlines expand into the outer solar atmosphere.

On the other hand, high-resolution observations suggest that the photospheric distribution of emerging magnetic flux in active regions can be interpreted in terms of multiple undulations. Pariat et al. (2004) applied a linear force-free extrapolation to these observational data and found that the EFR possessed a hierarchy of small undulating loops, overlaid by larger loops that may account for arch filament systems. The general geometry of the system consists of a series of connected $\boldsymbol{U}$ and $\Omega$ segments that form an overall "sea-serpent" configuration of an emerging field.

The "sea-serpent" topology was first suggested by Harvey \& Harvey (1973) to explain the undulating pattern of moving magnetic features near sunspots. Although there is no detailed explanation for this phenomenon yet, many observations are consistent with the "sea-serpent" model. Bernasconi et al. (2002) reported on the structure of moving dipolar features when new magnetic flux was emerging in an active region and showed that they appeared as undulations in the emerging field. They suggested that moving dipolar features are, in fact, $\boldsymbol{U}$-loops or flux ropes with a concave-downward shape that are tied to the photosphere by trapped mass. A process by which these fields could escape through the solar surface has been proposed by Spruit et al. (1987).
An interesting result of the work by Pariat et al. (2007) was that the undulatory magnetic flux system seemed to be connected to the photosphere by bald patches (BPs). BPs are associated with fieldlines having dips tangent to the photosphere and, thus, it is likely to be related with the $\boldsymbol{U}$ segments of the undulating field. In general, they are located over an inversion line $\left(B_{z}=0\right)$ where the magnetic field has a positive curvature $\left(\boldsymbol{B} \cdot \nabla B_{z}>0\right)$. If the same condition is satisfied for heights above the photosphere, then the region possesses a magnetic dip. The criterion for the existence of BPs was first given by Seehafer (1986) and later on, in more detail by Titov \& Démoulin (1999). Dipped magnetic field configurations have been studied by Mackay et al. (1999) and Mackay \& van Ballegooijen (2009).

Fieldlines that go through BPs belong to quasi-separatrix surfaces where current layers may develop, leading to occurrence of reconnection. Thus, BPs appear to be preferential sites of reconnection and possible sites of heating enhancement. Mandrini et al. (2002) found that some arch filament systems and surges, which were linked with flux emergence in a short-lived active region, were also associated with BPs. They also showed that current layers can be formed at BPs, detected in this active region, and efficiently dissipated in the chromosphere.

Pariat et al. (2004) studied the magnetic topology of serpentine-like emerging field lines and found that there is a close connection between BPs and the location of Ellerman bombs (EBs), which are small-scale brightenings and brief emissions in the wings of the $H_{\alpha}$ line. EBs were firstly observed by Ellerman (1917) and have been detected mainly within active regions. They have been observed in regions of emerging flux (between the two main polarities) and also in regions that surround isolated sunspots (Socas-Navarro et al. 2006).

The observations by Pariat et al. (2004) and Georgoulis et al. (2002) correspond to the central region of an emerging active region. Based on these observations Pariat et al. (2004) proposed a resistive flux emergence model where EBs are connected with 
low-lying fieldlines with a serpentine shape. Then, coronal loops in active regions and long flux systems (e.g. arch filament systems) may appear due to the rise of undulating magnetic fields that they reconnect at BPs. These loops get rid of the over-dense photospheric plasma by reconnection at BPs. The brightenings at the regions where reconnection occurs may account for the observational appearance of EBs (Ding et al. 1998; Georgoulis et al. 2002; Pariat et al. 2004; Fang et al. 2006). The undulatory shape of the rising magnetic field and the reconnection at the low atmosphere may also be used for the explanation of moving magnetic features and the EBs observed surrounding sunspots.

The wavelength of the undulations of the "sea-serpent" field has been found to be larger than $2 \mathrm{Mm}$. From a set of identified serpentine lines in their model, Pariat et al. (2004) found that the distance between successive EBs along the undulating flux tubes has a peak around $3 \mathrm{Mm}$ and a cutoff for values smaller than $2 \mathrm{Mm}$. Watanabe et al. (2008) reported that magnetic fields around EBs are undulatory with a characteristic wavelength of $\approx 4 \mathrm{Mm}$. These sizes are larger than the convective scales for granulation $(\approx 1 \mathrm{Mm})$ or less than supergranulation $(\approx 10 \mathrm{Mm})$ and are indicative of the presence of some instability.

The Parker instability (Parker 1966) may develop at the interface between the emerging flux system and the isothermal photosphere. The interface is subject to the instability when a perturbation of some wavenumber $\kappa$ is imposed on the lighter (compared to the surrounding plasma) but more magnetized flux system. For large wavenumbers, the instability is inhibited by the magnetic tension of the highly curved fieldlines. However, quantitative estimates have shown that there is a critical wavelength below which the magnetic tension is not able to prevent the triggering of the instability. One finds that, using a typical value for the temperature at the photosphere $(\approx 5800 \mathrm{~K})$, an undulating flux system will be Parker-unstable if the wavelength of the undulations is $\lambda>2 \mathrm{Mm}$. Nozawa et al. (1992) who studied the linear and non-linear properties of the Parker instability in a convectively unstable layer has confirmed the above results. Also, Shibata et al. (1989) performed 2D MHD simulations showing that a sub-photospheric horizontal flux sheet is unstable to the undular mode of the Parker instability and forms a rising magnetic loop that expands into the corona. The most unstable wavelength for the fastest growing mode is $\approx 20 H_{\mathrm{ph}}$, where $H_{\mathrm{ph}}$ is the pressure scale height at the photosphere.

The above theoretical considerations and observations suggest that undulations in a magnetic flux system may develop due to the Parker instability. The further emergence of the magnetic field is subject to the magnetic buoyancy instability and the process of reconnection along the serpentine field lines. The first numerical experiments, which were performed to study the evolution of an undulatory magnetic field due to the Parker instability were 2D and reported by Isobe et al. (2007). They found that magnetic reconnection in the lower solar atmosphere could explain the main characteristics of EBs, such as temperature and density enhancement. They also reported on the formation of long magnetic flux systems in the higher atmosphere and the formation of surge-like structures at low atmospheric heights due to reconnection.

In this paper, we present the results of the first threedimensional model of resistive flux emergence of an undulating flux system. We focus on two main aspects of the problem: firstly, the way the field is emerging into the corona, leaving the dense plasma in the low atmosphere, and forming largescale flux systems into the corona and secondly, how small-scale brightenings, with the characteristics of EBs, are triggered during the emergence of the field. We also report on other events such as the intensification of the photospheric magnetic field and new episodes of magnetic flux emergence.

The layout of the present paper is as follows: Sect. 2 presents the equations and the model used in the numerical experiments. Section 3 describes the initial phase of the rising motion of the emerging field at the photosphere. The distribution of the velocity field, the magnetic field and the plasma $\beta$ are studied. The further emergence of individual magnetic loops into the corona is discussed in Sect. 4.1. The global evolution of the magnetic field into the higher atmosphere is reported in Sect. 4.2. The resistive emergence of the field and the formation of Ellerman bombs are presented in Sect. 5. Section 6 presents events (such as magnetic field intensification and new emergence of magnetic flux) during the late stage of the evolution of the magnetic field. Finally, Sect. 7 contains a summary of conclusions and discussion.

\section{Model}

\subsection{Equations}

The initial setup of our experiment is similar to Isobe et al. (2007) with the main difference that we perform the numerical simulations in three dimensions. We solve the time-dependent, resistive and compressible MHD equations in Cartesian geometry. The basic equations (here in dimensionless form) are:

$\frac{\partial \rho}{\partial t}+\nabla \cdot(\rho \boldsymbol{u})=0$

$\frac{\partial(\rho \boldsymbol{u})}{\partial t}=-\nabla \cdot(\rho \mathbf{u} \boldsymbol{u})+\left(\frac{\nabla \times \boldsymbol{B}}{4 \pi}\right) \times \boldsymbol{B}-\nabla P+\rho \boldsymbol{g}$

$\frac{\partial}{\partial t}\left(\frac{P}{\gamma-1}+\frac{1}{2} \rho u^{2}+\frac{B^{2}}{8 \pi}\right)$

$+\nabla \cdot\left(\left(\frac{\gamma}{\gamma-1} P+\frac{1}{2} \rho u^{2}\right) \boldsymbol{u}+\frac{1}{4 \pi} \boldsymbol{E} \times \boldsymbol{B}\right)-\rho \boldsymbol{g} \cdot \boldsymbol{u}=0$

$\frac{\partial \boldsymbol{B}}{\partial t}-\nabla \times \boldsymbol{E}=0$

where

$\boldsymbol{E}=\eta \boldsymbol{J}-\boldsymbol{u} \times \boldsymbol{B}$,

$\boldsymbol{J}=\nabla \times \boldsymbol{B}$.

In the above equations, $\boldsymbol{E}, \boldsymbol{B}, \boldsymbol{J}$ and $\boldsymbol{u}$ are the electric field, the magnetic field, the current and the velocity field respectively. The gravitational acceleration is $\boldsymbol{g}=(0,0,-1 / \gamma), \eta$ is the resistivity and $\gamma$ is the ratio of specific heats. The medium is assumed to be an inviscid perfect gas with $\gamma=1.1$. Temperature is calculated from the equation of state as

$T=\gamma \frac{p}{\rho}$

Ohmic heating is taken into account. For the explicit resistivity we use a constant value of $\eta=0.001$. The magnetic Reynolds number $\left(R_{\mathrm{m}}=U \ell / \eta, U\right.$ and $\ell$ are characteristic velocity and length scales and $\eta$ is the magnetic diffusivity) in our simulations is $R_{\mathrm{m}} \approx 1000$. This is a typical value used in similar flux emergence experiments in three dimensions with a highly stratified atmosphere.

We use the same normalization method and units with Isobe et al. (2006). Our units (with typical values in the solar photosphere) are as follows: a pressure scale height of $H_{\mathrm{ph}}=180 \mathrm{~km}$ 

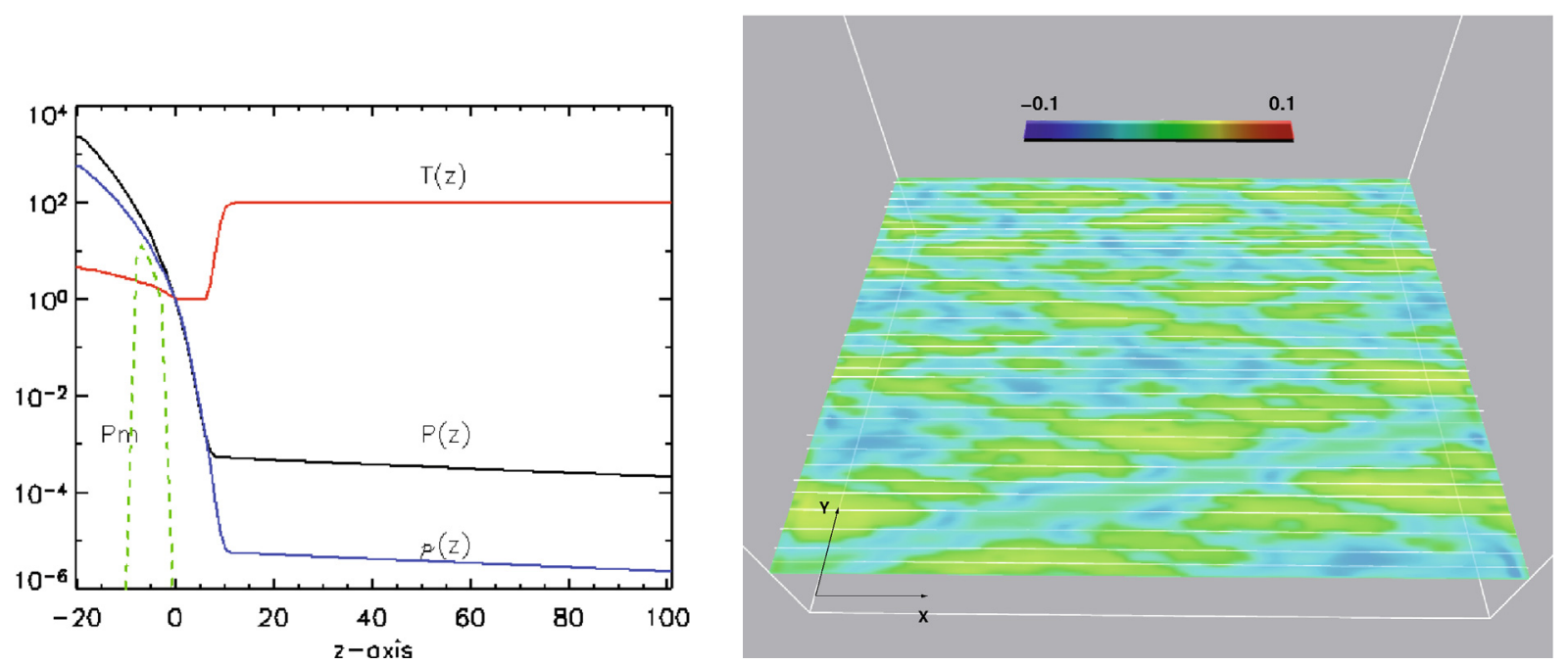

Fig. 1. Left: the initial stratification of the background atmosphere $(t=0)$. Right: the coloured contours show the initial distribution of the vertical component of velocity at the $x y$ plane at $z=-5$. The horizontal slice extends across the whole numerical domain in the $x$ - and $y$-direction.

for the length, $u_{\mathrm{ph}}=9 \mathrm{~km} \mathrm{~s}^{-1}$ for the velocity and $t_{\mathrm{ph}}=20 \mathrm{~s}$ for time. We also use $T_{\mathrm{ph}}=6000 \mathrm{~K}$ for temperature, $\rho_{\mathrm{ph}}=$

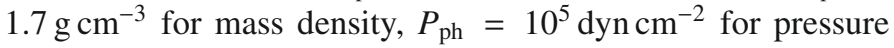
and $B_{\mathrm{ph}}=300 \mathrm{G}$ for the magnetic field strength. Hereafter, all the plots presented in this paper are dimensionless (one has to multiply by the above units to estimate the physical time and length scales in the plots).

The above equations were numerically solved by using the CANS (Coordinate Astronomical Numerical Softwares) code, with a modified Lax-Wendroff scheme and artificial viscous dissipation.

\subsection{Initial conditions}

We use numerical simulations to study the emergence of magnetic flux from the upper convection zone into the corona. The initial background atmosphere consists of four layers, which are in hydrostatic equilibrium. The upper convection zone is represented by a superadiabatically stratified layer in the range $(-20 \leq z<0)$. An isothermal layer at $0 \leq z \leq 5$ represents the photosphere. The layer above the photosphere $(5<z<12$ is mimicking the chromosphere/transition region. The uppermost layer $(12 \leq z \leq 80)$ is an isothermal layer that represents the corona. The initial distribution of the temperature is:

$T(z)=T_{\mathrm{ph}}-\left(\alpha\left|\frac{\mathrm{d} T}{\mathrm{~d} z}\right|_{\mathrm{ad}}\right) z$

for $-20 \leq z \leq 0$. The adiabatic temperature gradient is:

$\left|\frac{\mathrm{d} T}{\mathrm{~d} z}\right|_{\mathrm{ad}}=\frac{\gamma-1}{\gamma} \frac{T_{\mathrm{ph}}}{H_{\mathrm{ph}}}$

and $\alpha$ is a dimensionless constant:

$\alpha=-\frac{\mathrm{d} T}{\mathrm{~d} z} /\left|\frac{\mathrm{d} T}{\mathrm{~d} z}\right|_{\mathrm{ad}}$.

For a convectively unstable layer $\alpha$ is larger than one, as predicted by the Schwarzchild criterion. In our simulations we use $\alpha=2$ for the sub-photospheric layer. Thus, any small velocity perturbation imposed on this layer may lead to instabilities due to the superadiabaticity of this layer.
For the atmosphere above the convectively unstable layer, the temperature distribution is:

$T(z)=T_{\mathrm{ph}}+\left(T_{\text {cor }}-T_{\text {pho }}\right)\left(\frac{1}{2}\left[\tanh \left(\frac{z-z_{\text {tr }}}{w_{\text {tr }}}+1\right)\right]\right)$

where we consider $T_{\mathrm{ph}}=1(\approx 6000 \mathrm{~K})$ for the temperature at the photosphere and $T_{\text {cor }}=100$ for the temperature in the corona. The mid height of the transition region is $z_{\mathrm{tr}}=11$ and the width of the transition region is $w_{\mathrm{tr}}=0.6 H_{\mathrm{ph}}$. Figure 1 (left panel) shows the initial distribution of the gas pressure $(P)$, temperature $(T)$ and density $(\rho)$ along height.

For the initial magnetic field we consider a horizontal and uniform magnetic field distribution, located within the upper levels of the unstable layer below the photosphere. Initially, the magnetic flux sheet is oriented along the positive $x$-direction. Thus, the magnetic field strength is:

$\boldsymbol{B}=\left(B_{x}, 0,0\right)$

where

$B_{x}=\sqrt{\left(\frac{8 \pi P}{\beta}\right)}$

where $\beta$ is the ratio of gas presure to magnetic pressure (plasma beta) and is given by:

$\frac{1}{\beta}=\frac{1}{\beta_{\mathrm{fs}}}\left(\frac{1}{2} \tanh \frac{z-z_{\mathrm{fsl}}}{w_{\mathrm{fs} l}}+1\right) \times\left(\frac{1}{2}\left(-\tanh \frac{z-z_{\mathrm{fsu}}}{w_{\mathrm{fsu}}}+1\right)\right)$.

Similar to the 2D experiments by Isobe et al. (2007), we take the plasma beta at the flux sheet $\left(\beta_{\mathrm{fs}}\right)$ to be equal to 4 . We also take the lower boundary of the flux sheet to be at $z_{\mathrm{fs}}=-10$ and the upper boundary at $z_{\mathrm{fsu}}=-1 . w_{\mathrm{fsl}}$ and $w_{\mathrm{fsu}}$ are the scale heights of the magnetic flux sheet at the lower and upper boundary and $w_{\mathrm{fsl}}=w_{\mathrm{fsu}}=0.5$ in our calculations. In Fig. 1 (left panel), $P_{\mathrm{m}}$ shows the distribution of the magnetic pressure in the sub-photospheric layer as a dashed curve. Clearly, $\beta$ is greater than unity.

Using Eqs. (6), (12) and (13), we can derive the distributions of density, the gas pressure and the magnetic field strength in the flux sheet by solving the equation of magnetostatic balance:

$\frac{\mathrm{d}}{\mathrm{d} z}\left(P+\frac{B^{2}}{8 \pi}\right)+\rho g=0$ 
Initially, a small velocity perturbation is imposed on the magnetic flux sheet, to excite the convective Parker-instability. The component of the velocity perturbation along the flux sheet has the form:

$$
\begin{aligned}
v_{x}= & A\left(\cos \left(2 \pi \frac{x}{\lambda_{\mathrm{p}}}\right)\right) \times\left(\frac{1}{2} \sin \left(2 \pi \frac{y}{\lambda_{\mathrm{p}}}\right)\right) \\
& \times \frac{1}{2}\left(\tanh \left(\frac{z-z_{\mathrm{fsl}}}{0.5}\right)-\tanh \left(\frac{z-z_{\mathrm{fsu}}}{0.5}\right)\right)
\end{aligned}
$$

where we take a small amplitude, $A=0.05$ and a wavelength, $\lambda_{\mathrm{p}}=20$, which is nearly the most unstable wavelength of the Parker instability in the linear phase. The $v_{y}$ and $v_{z}$ components have a similar distribution to $v_{x}$. Thus, the initial velocity perturbation is three-dimensional and non-uniform with a small amplitude. The right panel in Fig. 1 shows the distribution of the vertical component of the velocity field at $z=-5$ and $t=1$. The horizontal (white) fieldlines show the topology of the initial magnetic field configuration. Eventually, the resulting $B_{z}$ will take positive and negative values on the $x y$-plane.

For the computational domain, we assume periodic boundary conditions for the $x$ and $y$ directions. At the lower boundary at $z=z_{\min }$ the boundary is symmetric (rigid conducting wall). At the top boundary $\left(z=z_{\max }\right)$ the $z$ derivatives of all variables are set to zero. We also include a wave damping zone for $z>65$ where fluctuations of velocity, gas pressure and density decay exponentially with time.

The physical size of the numerical box is $0 \leq x \leq 100$, $0 \leq y \leq 80$ and $-20 \leq z \leq 80$. The number of the grid points are $N_{x}=400, N_{y}=140$ and $N_{z}=200$. The grid spacing is uniform in the horizontal directions, $\Delta_{x}=0.25$ and $\Delta_{y}=0.57$. In the $z$ direction $\Delta_{z}=0.5$ for $z \leq 40$, while it increases for larger heights.

\section{Emergence into the photosphere}

\subsection{Rising magnetic field and velocity pattern.}

Due to the initial velocity perturbation and the convectively unstable layer below the photosphere, a number of magnetic loops are formed out of the initial magnetic flux sheet. The magnetic field develops a number of undulations and adopts an overall serpentine-like shape. Initially, the perturbed segments (looplike structures) of the magnetic flux sheet rise due to the Parker instability. The size of the undulations and the draining of the plasma along the fieldlines at the crests of the rising loops are characteristic of the Parker instability. As the instability develops, the plasma slides down along the magnetic field, enhancing the magnetic buoyancy of the rising loops.

The instability in the sheet is excited by the initial velocity perturbation. The characteristic wavelength in the magnetic field that appears during the dynamical evolution of the system is between $10 H_{\mathrm{ph}}$ and $20 \mathrm{H}_{\mathrm{ph}}$. Notice that the Parker instability in an isothermal gas has its maximum growth rate for a wavelength in the range $10 H_{\mathrm{ph}}<L<20 H_{\mathrm{ph}}$. Now, the evolution of the convective-Parker instability is determined by modes with large wavelength, independent of the initial perturbation wavelength. The perturbation with the most unstable wavelength is efficiently created by nonlinear mode coupling (Nozawa et al. 1992). Modes with small wavelength are stabilized due to the downward magnetic tension of the fieldlines.

Figure 2 shows the evolution of $v_{z}$ at $z=1$ for $t=20,30,40$ and 50. As time goes on, the velocity field loses its initial random configuration and consists of a network of cells that have upflows in their middle section. At the boundaries between the cells, there are pronounced downflows. At $t \approx 50$, the size of the cells varies from $10 H_{\mathrm{ph}}$ to $30 H_{\mathrm{ph}}$. This network of upflows and downflows forms a pattern, which evolves dynamically in space and is highly time-dependent. The deformation of the initial velocity field is due to the emergence of the sub-photospheric magnetic field. The upflows correspond to the upward rising motion of the magnetic field and the downflows occur at the periphery of the emerging loop-like structures as the plasma drains down along the fieldlines of the loops.

The initial appearance of the magnetic loops is shown by the the magnetic fieldlines, which are overplotted in the four panels of Fig. 2. The fieldlines have been traced from random points at the base of the photosphere. At $t=20$, they are almost straight and they are oriented along the $x$-axis. Eventually $(t=30$ and $t=40$ ), some segments are rising forming loop-like structures that eventually emerge through the photosphere. There is a good comparison between the location of the upflow regions and the sites of rising loops. Also the downflows appear in between the rising loops and become stronger as the loops rise higher into the atmosphere $(t=50)$. Also, notice that now the magnetic fieldlines are not oriented only along the $x$-axis but they can follow various directions in the three-dimensional volume. This is due to various reasons: one is the shearing that some fieldlines experience due to the initial velocity perturbation, a second reason is the appearance of interchange modes due to the strong non-linear coupling of the magnetic field. At later times, due to the three-dimensional expansion of the field, neighbouring fieldlines may reconnect and change their connectivity. The initial evolution of the magnetic field distribution at the photosphere is discussed in more detail in the next section.

\subsection{Distribution of magnetic field and plasma $\beta$.}

The panels in the left column of Fig. 3 show the distribution of the vertical component of the magnetic field $\left(B_{z}\right)$ at low photospheric heights $(z=1)$, for $t=50, t=60$ and $t=70$. The distribution reveals a network consisting of mixed, positive and negative flux sources scattered across the photosphere. A comparison between Figs. 2 (bottom-right panel) and 3 (topleft) shows that the regions with relatively large values of $B_{z}$ at the photosphere are located at the sites where the undulations of the fieldlines pierce the photospheric layer. In fact, the magnetograms of Fig. 3 show the horizontal cross-section of the looplike magnetic field structures that rise into the photosphere. From $t=50$ to $t=70$ the appearance of $B_{z}$ at the photosphere becomes more pronounced because the inclination of the legs of the rising loops becomes more vertical. The latter is due to the efficiency of buoyancy developed by the Parker instability. Also, the absolute value of $B_{z}$ increases during the evolution, as more magnetic layers from the sub-photospheric layer emerge into the photosphere. The intensification of the magnetic field at the low atmosphere is also discussed in Sect. 6.1.

The evolution of the magnetic field distribution shows, that although the network of mixed polarities appears to be complex, there is an order on the appearance of the flux sources. Positive polarities appear at the left side of the rising loops and move leftward while negative polarities appear on the right side of the emerging fields and move towards the right side of the numerical domain. The apparent order on the initial flux distribution is mainly due to the fact that the original flux sheet is uniform and orientated along the $x$-direction.

Overplotted is the horizontal velocity field (arrows) onto the horizontal plane. The velocity distribution shows that a) there is 

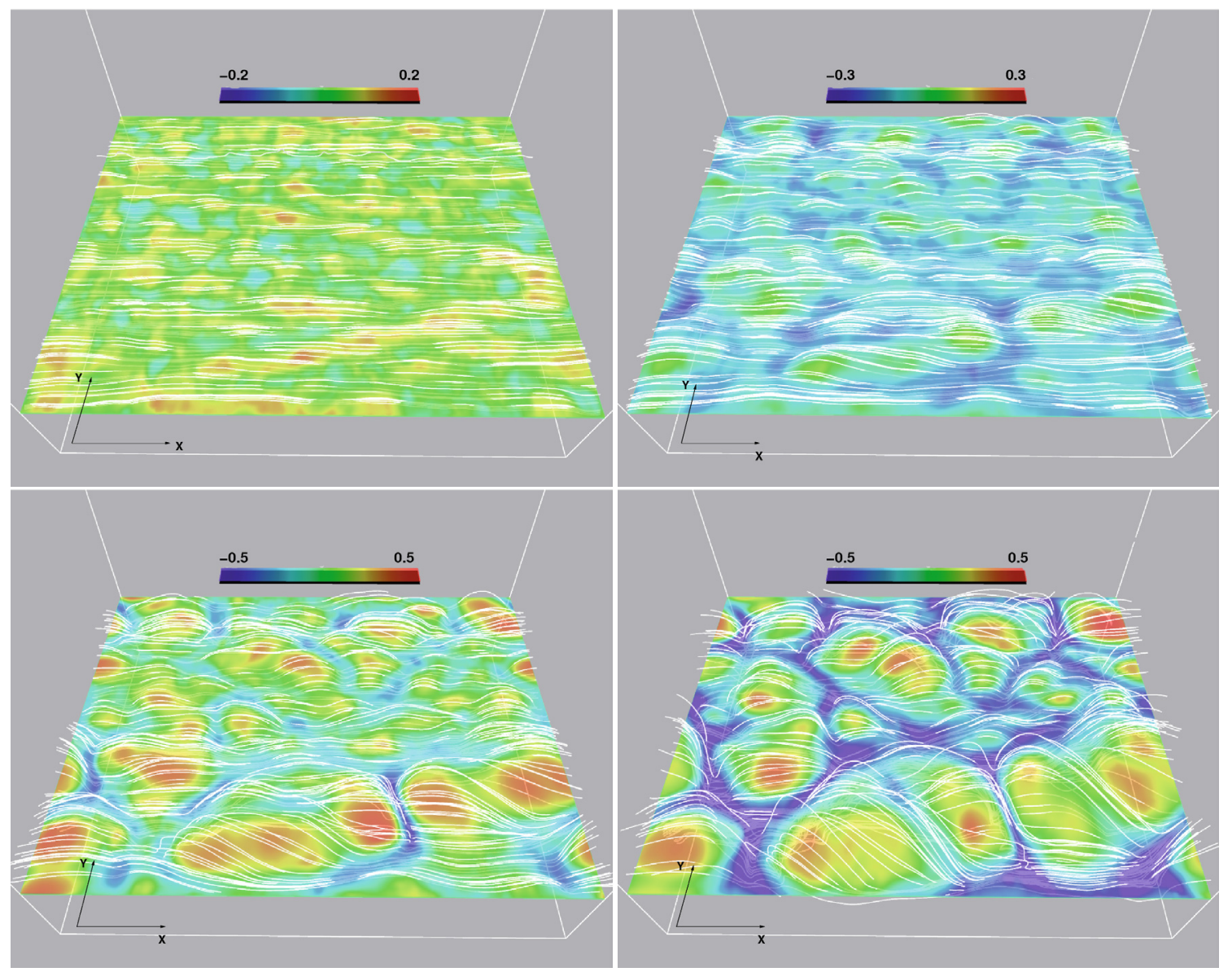

Fig. 2. The distribution of $v_{z}$ at the photosphere for $t=20,30,40$ and 50 (from up-left to bottom-right). The horizontal planes extend across the whole numerical domain in the $x$ - and $y$-direction.

a general diverging flow as the two opposite polarities stream away from the central region of the emerging loops and b) there are converging motions at the internetwork, at the boundaries between the emerging loops. This is due to the expansion of the rising magnetic field and the downflows from the crest to the periphery of the three-dimensional loops.

The apparent horizontal motion of the migrating polarities may reach speeds of about $1-2 \mathrm{~km} \mathrm{~s}^{-1}$. As they drift apart, flux sources may experience encounters with opposite-polarity fields. These flux cancellation events are common, especially during the advanced stage $(t>70)$ of the evolution of the system, leading to a general decrease of the total unsigned flux at the photosphere.

In the right column of Fig. 3, the $\log (1 / \beta)$ is shown for the same height $(z=1)$ and times. We find that at $t=50$, the rising magnetic field has just crossed the isothermal photosphere. The magnetic loops experience horizontal expansion but they cannot proceed with further emergence into the outer atmosphere. After $t \approx 55$ plasma $\beta$ is reduced, as more field rises from the interior to the photosphere, and obtains values smaller than one in various regions across the photosphere. The build-up of the magnetic pressure at these regions is associated with the increase of $B_{z}$ and the emergence of more magnetic flux from the solar interior as we discussed above.

As the plasma $\beta<1$, the magnetic pressure exceeds the gas pressure and the first flux elements begin to rise and expand above the photosphere. Similar to the work by Archontis et al. (2004), we find that as plasma $\beta<1$, the evolution occurs on the basis of the magnetic buoyancy instability experience by the plasma above the photosphere. More precisely, we find that the strong stabilizing term $-(\gamma / 2) \beta \delta$ ( $\delta$ being the superadiabaticity of the atmosphere) adopts small values and actually becomes smaller than $-H_{\mathrm{ph}}(\partial / \partial z) \log B$. The latter term describes the instability-promoting effect of the decrease of the magnetic field with height in the upper part of the rising magnetic field.

By $t=70$, stronger field has reached the photosphere and, thus, plasma $\beta$ is reduced significantly. The distribution at the photosphere shows a sporadic decrease of plasma $\beta$ : at this stage of the evolution the magnetic flux sheet does not emerge as a whole structure but it rather consists of individual magnetic loops that rise due to their own magnetic pressure force. The emergence of the magnetic loops into the corona is discussed in the next section.

\section{Emergence into the corona}

\subsection{Local evolution of the magnetic field}

To study the emergence of the undulating magnetic field into the corona we follow first of all the rising motion of one magnetic loop, which is part of the large-scale magnetic flux system. We focus our attention on regions at the photosphere where plasma $\beta$ is small and, thus, magnetic pressure is large. One of the regions is located at $60<x<80$ and $45<y<60$ (see middle-left panel in Fig. 3). The magnetic field meets the photosphere in this region and eventually forms an $\Omega$-loop shape that rises into the corona. Figure 5 (top panel) shows the temporal evolution of the height of the apex of this loop. More precisely, we plot 

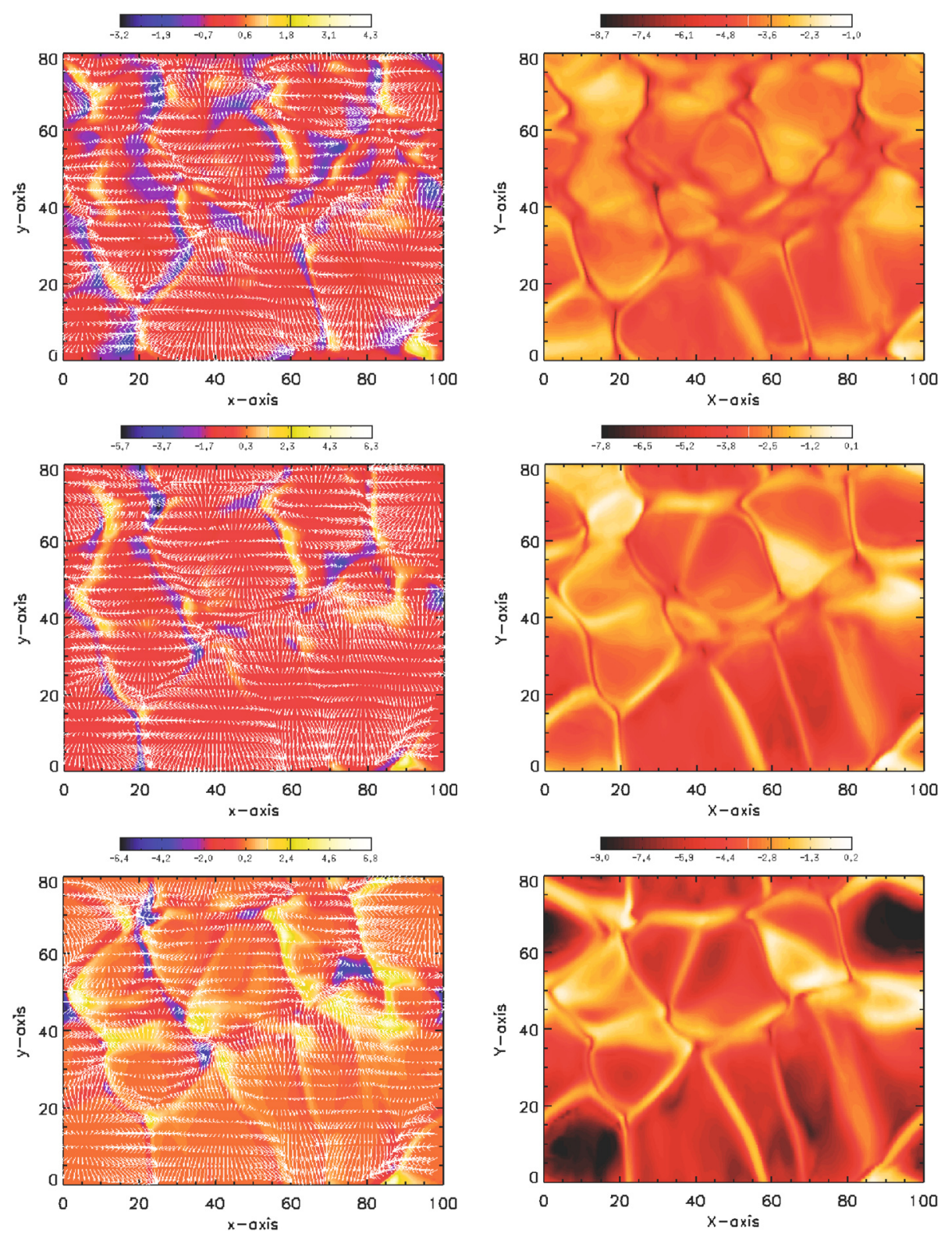

Fig. 3. Left: distribution of $B_{z}$ at $z=1$ for $t=50, t=60$ and $t=70$. The horizontal velocity distribution is shown with the overplotted arrows. Right: the same, for the distribution of $\log \left(\frac{1}{\beta}\right)$.

the height of the apex at $x=65$ and $y=52$. The magnetic field strength is weak at the top of the rising loops and, thus, we define the apex as the point where the magnitude of $\boldsymbol{B}$ is $\approx 10^{-4}$. A sideview visualization of this loop together with its rising motion through the atmosphere is shown in the next section.

The emergence starts when the magnetic field experiences the upward forcing of the initial velocity perturbation. The apex of the magnetic loop reaches the photosphere at $t \approx 20$. The further upward motion involves lifting up dense plasma against gravity, within the photosphere that is stable against the convective instability. Thus, the loop continues to rise through the photosphere but with small velocity and considerable expansion in the transverse direction (see also bottom panels in Fig. 3). After $t=50$, plasma $\beta$ drops sufficiently so that the evolution of the magnetic field occurs due to the buoyancy instability as has been described in Archontis et al. (2004) and Murray et al. (2006). Now the magnetic field is emerging all the way up into the corona and experiences a runaway expansion.

Figure 5 (bottom panel) shows the distribution of the magnetic and the gas pressure along height, within the emerging loop. At $t=80$, the apex of the loop has reached a height just above $z=20$ and, thus, it is well inside corona. Notice that the magnetic pressure (solid line) is larger than the gas pressure (dashed line) inside the rising, magnetized volume of the field and, thus, the plasma is free to expand as it rises into the higher atmosphere.

\subsection{Global evolution of the magnetic field.}

We now study the global evolution and rise of the magnetic field into the corona. Figure 4 consists of four panels that show the emergence of the magnetic field at four different times. The coloured contours on the transparent vertical slice $(y=52)$ show the magnitude of the magnetic field strength $|\boldsymbol{B}|$. The horizontal slice shows the vertical component of the magnetic field, $B_{z}$, just above the base of the photosphere $(z=1)$. The fieldlines are traced from the vertical slice, at points within the emerging field.

At $t=60$ (top panel in Fig. 4), the initial magnetic flux sheet has developed a number of loops (L1 to L4), which are interconnected with serpentine-like fieldlines ( $u 1$ and $u 2$ ). From the distribution of $B_{z}$ at photospheric heights, we find that the dips of the fieldlines are located at regions between the positive and 

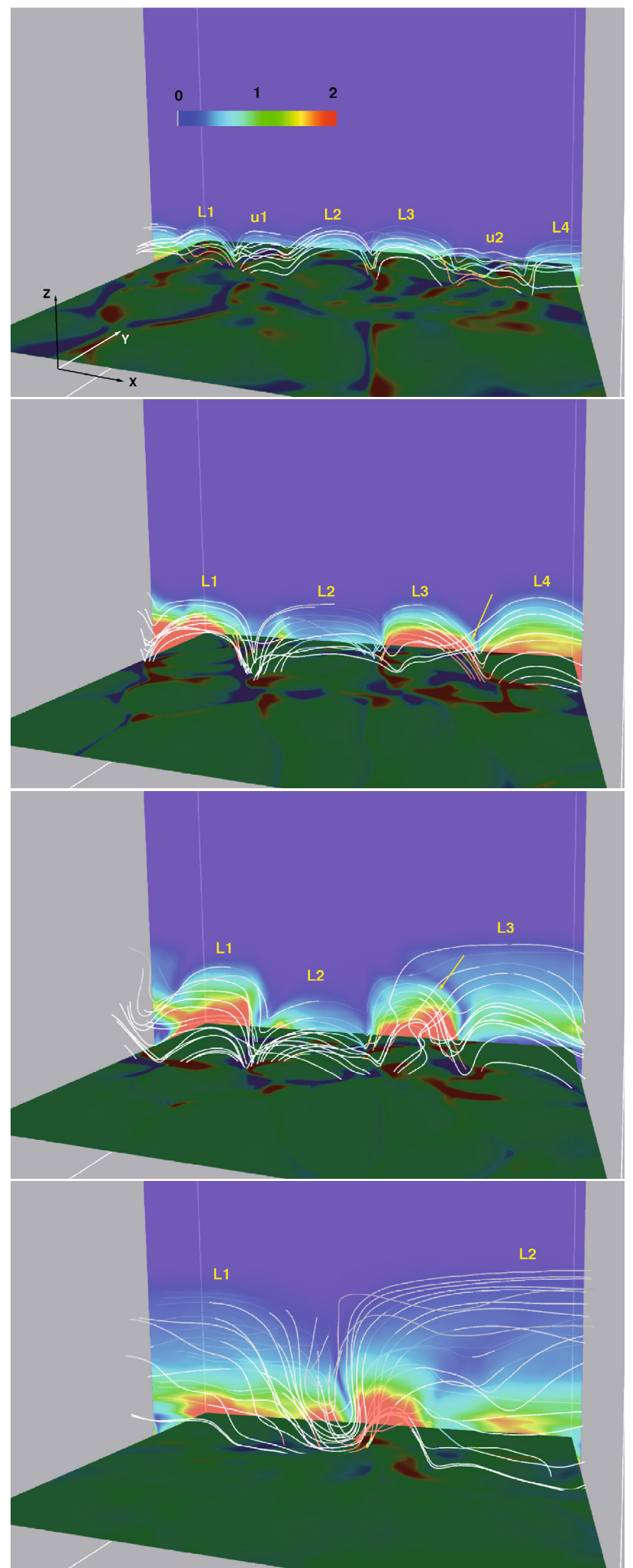

Fig. 4. Evolution of the magnetic field strength for $t=60,70,90$ and $t=130$. The vertical slice shows the distribution of the total magnetic field strength at $y=71$. The horizontal slice shows the $B_{z}$ distribution at the base of the photosphere. The range of values for $B_{z}$ is -2 to 2 . The horizontal and vertical planes extend across the whole numerical domain, in the $x-y$ and $x-z$ directions respectively.
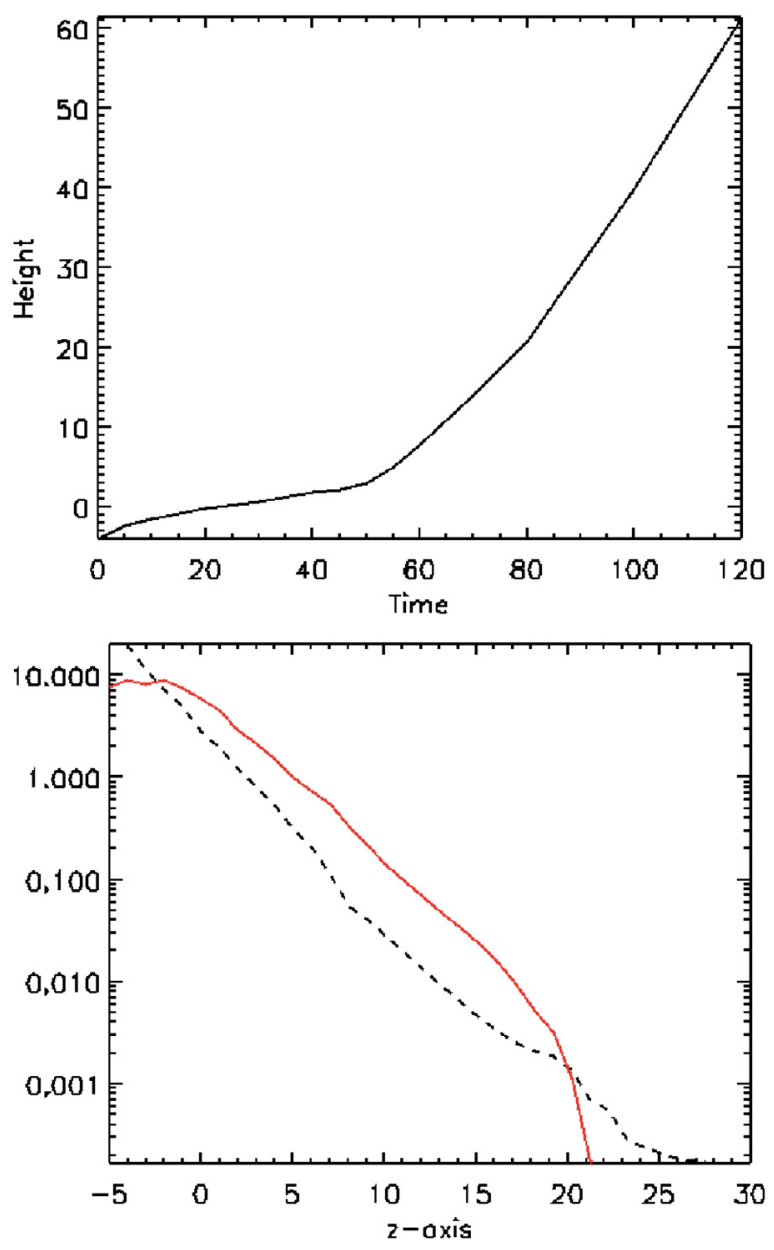

Fig. 5. Top: height-time profile of a loop that is emerging into the corona. Bottom: distribution of gas pressure (dashed line) and magnetic pressure (solid line) along height (within the emerging loop), at $t=80$.

negative polarities of neighbouring loops. Some of the dips cross the photosphere and have their lowest concave part below the photosphere, while other dips are tangent to the photosphere at BPs. Most of the loops have entered the chromosphere/transition region but they have not yet expanded dynamically into the corona. Examples of emerging loops are loops L2 and L3, at the center of the vertical slice. The evolution of the central-right loop (L3) was studied in the previous section. Notice, that on the two sides of the loops L2 and L3 there are segments of fieldlines that do not form discrete, coherent loops, which rise dynamically into the atmosphere. These fieldlines undergo loose undulations at low photospheric heights (see $\mathrm{u} 1$ and $\mathrm{u} 2$ ). This is the typical topology of the magnetic field at this stage of the evolution: a network of emerging loops and low-lying undulating fieldlines, which are joined across the photosphere.

At $t=70$, the visualization of $|\boldsymbol{B}|$ at the vertical slice shows the formation of four main magnetic loops across the $x$-direction. The two central loops continue to rise into the atmosphere. They also expand sideways as they rise. Due to the expansion, neighbouring fieldlines approach to each other and reconnect. Reconnection occurs between fieldlines with opposite directions that come into contact at the vicinity of the dips. Due to successive reconnection at various heights, longer loops without pronounced undulations may form during the dynamical evolution of the system. One example of this process is the formation of the two loops (L3 and L4) at the right side of the computational 
volume. Reconnection occured between these loops and the undulating fieldlines $(\mathrm{u} 2)$ in the spare region between the loops at earlier times (e.g. after $t=60$ ). As a result, longer loops have been formed, which in turn, they will come closer together and reconnect at later times. This is similar to the process of resistive flux emergence, which has been presented in theoretical grounds by Pariat et al. (2004) and in numerical experiments by Isobe et al. (2007).

Indeed, at $t=90$ there are reconnected fieldlines that join the crests of the two loops L3 and L4 (seen at $t=70$ ) forming a new, longer flux system L3 (with a typical loop-like shape and a length of about 9-10 Mm), which has emerged well into the corona. Also, some of the low-lying fieldlines that appear at the central part of the domain at $t=70$ and $t=90$, start from the positive polarity of the far-left magnetic loop (L1) and they end up in the crest of the right loop (L4). Actually, these fieldlines join all the loops without going through BPs. These are also reconnected fieldlines for which all of their segments start to rise above the photosphere and, eventually, into the outer atmosphere.

At $t=130$, most of the magnetic fieldlines that previously enveloped the emerging loops have now been reconnected at and above photospheric heights, forming two long magnetic flux systems (L1 and L2) that reach heights around $z=65$. At this specific time and position along the $y$-direction, there is only one discernible U-shaped dip at the center of the domain where the fieldlines touch the low atmosphere. Later on, this U-dip will be diminished when local reconnection events will join the two lateral magnetic systems.

It is worthwhile mentioning that the fieldlines that belong to the two flux systems (L1 and L2) have different shapes at different heights. Fieldlines, which are lying at low heights underneath the large expanding outer loops, still possess undulations along their lengths. This topology is an indication of reconnection that occurs at different heights during the process of flux emergence. With this mechanism, the large reconnected fieldlines get detached from the low atmosphere and are free to rise into the corona. The evolution of the magnetic field described in this section is typical of the global magnetic field in this numerical experiment.

\section{Resistive emergence and Ellerman bombs}

One question that arises from the resistive flux emergence model is the role of reconnection on the emergence of the magnetic flux system into the higher atmosphere. Our simulation show that reconnection occurs firstly at the photosphere when the sufficiently long flux sheet becomes subject to the Parker instability, developing a series of undulations.

Figure 6 is a closeup of the magnetic reconnection at the low atmosphere. The size of the part of the domain shown in this figure is $60 \leq x \leq 70, y=30$ and $-11 \leq z \leq 7$. The horizontal black line shows the base of the photosphere. The arrows show the direction of the full velocity vector and the white lines are the magnetic field lines, which are traced from the flanks of the two magnetic loops into contact. The two loops shown in this figure are a small part of the whole undulating magnetic field. At $t=50$ (top panel), there are converging downflows at the flanks of the loops due to the rising motion of the magnetic field. The magnetic fieldlines form a U-dip at the interface. In fact, these dips are sites where dense plasma is trapped as it is drained down by the flows, along the flanks of the emerging field. Since the temperature gradient in the sub-photospheric

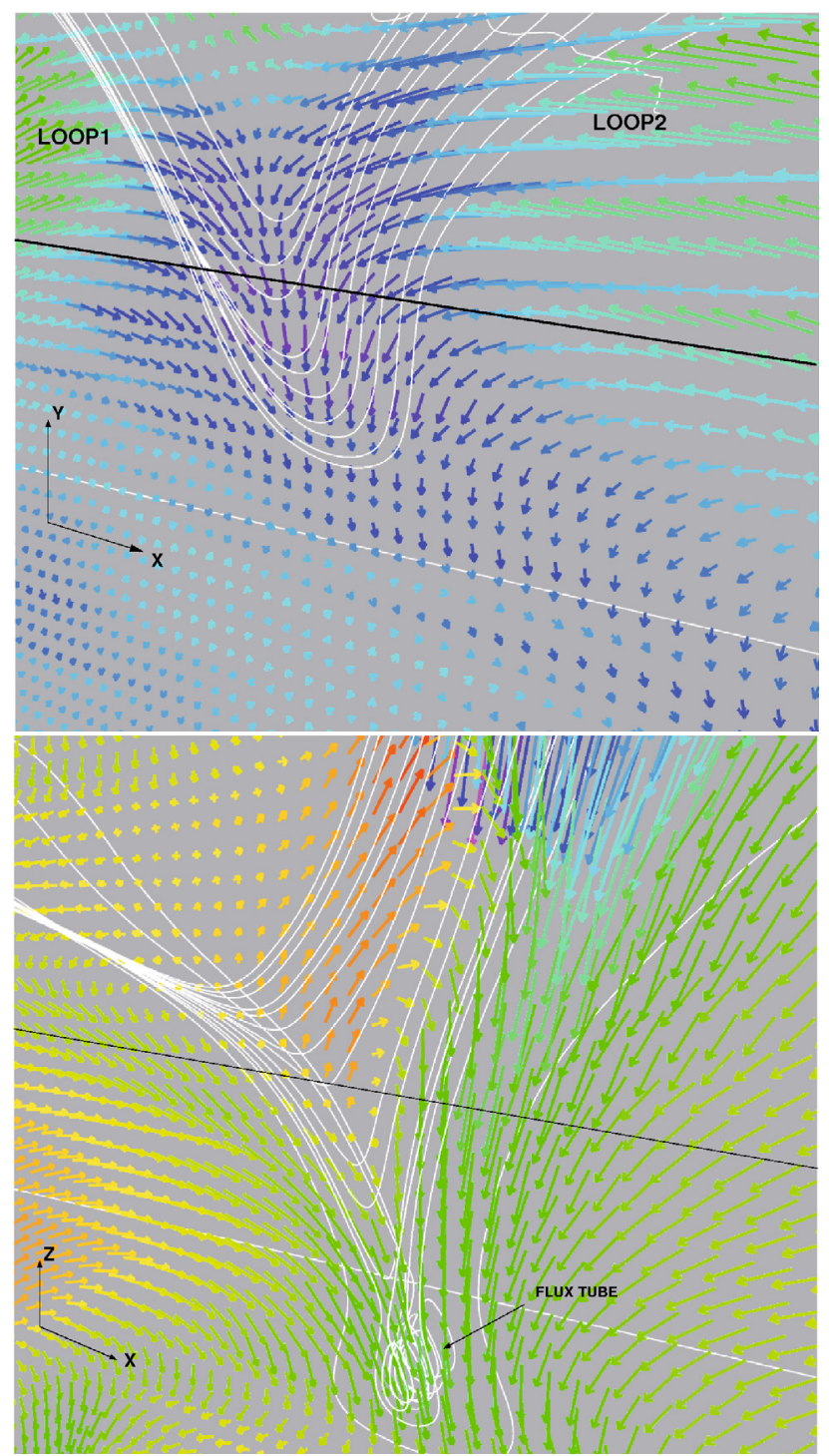

Fig. 6. Top: close-up in one of the dips that magnetic fieldlines (white lines) possess along the undulating magnetic flux system at $t=50$. The arrows show the direction of the full velocity vector. Bottom: the same location but at a later stage of the evolution of the system $(t=60)$. The color of the arrows is changing from dark blue (negative values, downflows) to red (positive values, upflows).

layer is superadiabatic, the concave part of the magnetic flux system sinks rapidly and, thus, induces converging flows towards the interface and enhances the downflows along the interface. When the oppositely directed fieldlines of the magnetic loops come close together, a current layer builds up at their interface. There is an average number of twelve grid points across the current layers, which are formed in the computational volume during the evolution of the system. Reconnection starts to occur at the current layer, producing bidirectional flows $(t=60$, bottom panel). In this regime, the flow is upward at the photosphere with a value of $\approx 1 \mathrm{~km} \mathrm{~s}^{-1}$. There is also a downflow, which is moving mainly within the sub-photospheric layer, with smaller velocity of about $0.2 \mathrm{~km} \mathrm{~s}^{-1}$.

Reconnection also changes the magnetic topology around the reconnection site. The dense plasma that is trapped in the U-dips stays below the photosphere in new reconnecting fieldlines, which are twisted and all together adopt the shape of small flux tubes. Higher up, the fieldlines that were previously linked 

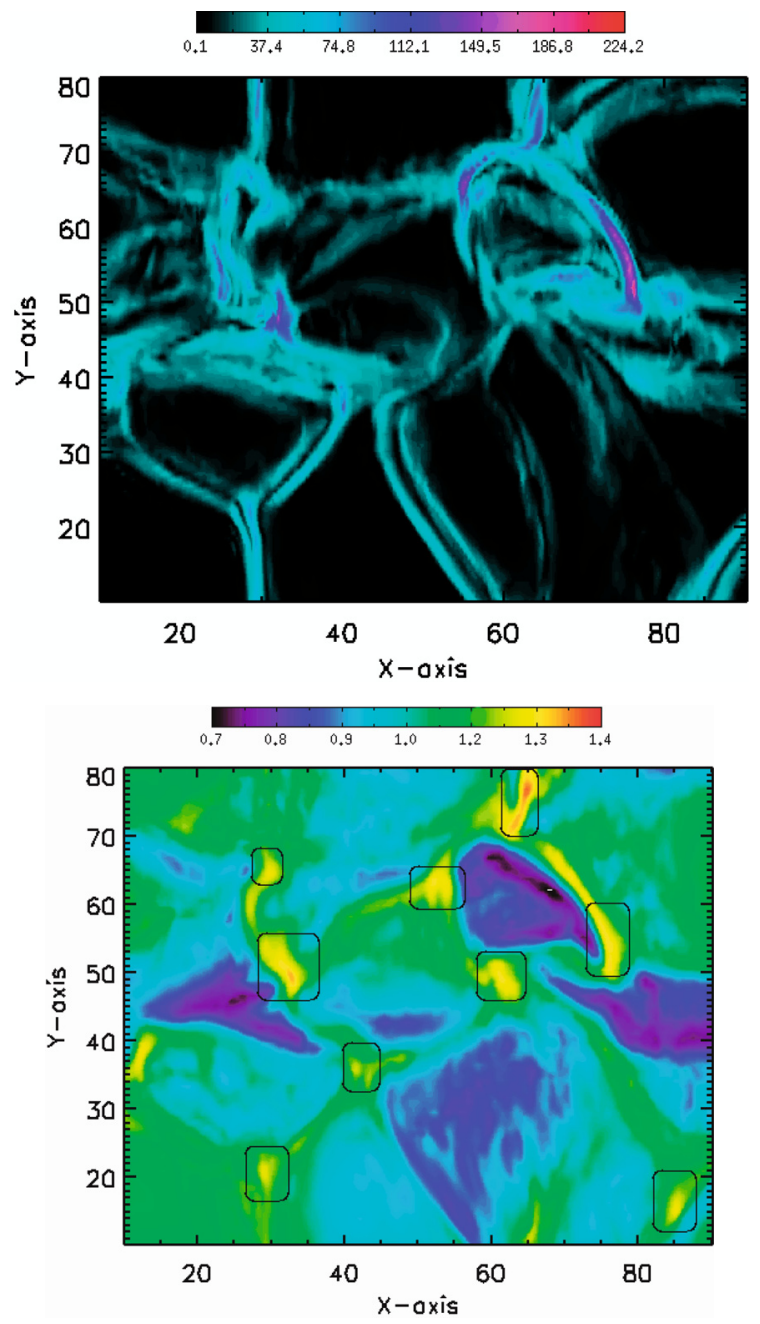

Fig. 7. Top: distribution of the total current density. Bottom: distribution of temperature. Both are taken at $z=6$ (low chromosphere) and for $t=90$.

to the photosphere, have now been reconnected and are rising into the higher atmosphere. The new reconnected fieldlines can envelope the two loops from above, and thus, connect the two far ends of the loops. This is a typical example of how reconnection works between neighbouring magnetic loops at this stage of the evolution of the system. By this process the magnetic field is getting rid of the dense plasma and gradually it rises through successive atmospheric heights.

As we discussed in the previous section, reconnection occurs at various heights of the atmosphere due to the three-dimensional emergence of the magnetic loops and to the non-linear and highly dynamic evolution of the system. Figure 7 shows the distribution of the current density $(\boldsymbol{J}=\nabla \times \boldsymbol{B})$ and the temperature at the low chromosphere $(z=6)$ at $t=90$. There are many current layers, which are formed at the interface between flux sources with opposite polarities. The flux sources belong to different loops that emerge and approach to each other. In some of these sites the current density is especially strong. For example, three sites with pronounced currents are the regions around $x=75, y=50, x=32, y=48$ and $x=55, y=65$. From the temperature distribution, we find that there is a good correlation between sites with strong current density and enhanced temperature. Notice, that the cool regions correspond to the plasma that expands adiabatically as the loops emerge.
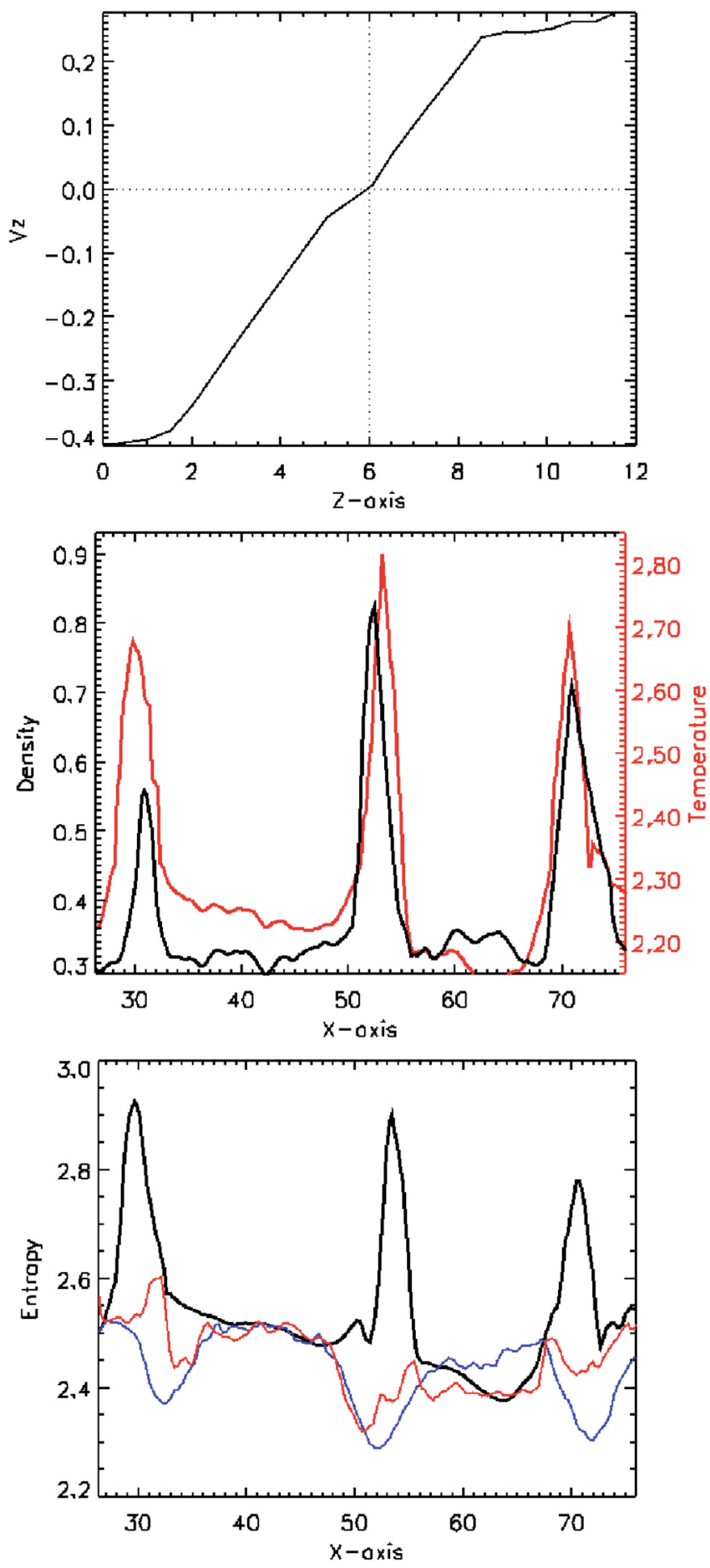

Fig. 8. Top: bi-directional flow that is heated due to reconnection at the low chromosphere. Middle: distribution of temperature and density along the $x$-direction at the low chromosphere for $y=65$ and $t=90$. Bottom: distribution of entropy along the $x$-direction at the low chromosphere for $y=65$ and $t=70$ (blue), $t=80$ (red) and $t=90$ (black).

The enhanced temperature is due to effective reconnection that occurs at the sites with strong current layers. As a result of reconnection, bi-directional flows are produced at the chromosphere. Figure 8 shows the reconnection outflow, which is produced at $t=90$ in the region around $x=55$ and $y=65$. The change in the direction of the outflows occurs at $z \approx 6$. Thus, at this stage of the evolution, the reconnection site is at the low chromosphere. The upflow is emitted within the chromosphere 
and the downflow towards the photosphere. According to our units, the upward velocity of the bi-directional flow in the chromosphere/transition region could be as high as $V_{z} \approx 2.2 \mathrm{~km} \mathrm{~s}^{-1}$ and the downflow could reach values up to $V_{z} \approx 3.6 \mathrm{~km} \mathrm{~s}^{-1}$. It is worth mentioning that in a few cases the upward outflows may reach velocities up to $20 \mathrm{~km} \mathrm{~s}^{-1}$, as the hot plasma is ejected above the reconnection site. These high speed outflows may account for surge-like features.

We are now looking at some of the properties of the magnetized plasma that is emitted due to reconnection that occurs in various locations within the rising magnetic flux system. Such locations are shown in Fig. 7 (marked with rectangular boxes). The horizontal profiles (along the $x$-direction) of temperature and density at $z=6, y=65$ and $t=90$ are shown in Fig. 8. The distribution shows that the temperature is enhanced in three regions by a factor of 1.3 , relative to the temperature of the initial background atmosphere at this height. The density distribution shows a good correlation with the temperature. Density takes large values at $x \approx 30, x \approx 52$ and $x \approx 71$. The maximum value shows that the density increases by a factor of 2.7 , relative to the density of the background plasma. The values of the relative enhancement of the temperature and density of the plasma may vary at different sites of reconnection. As an example, the density enhancement could reach a factor of 4 at this height during the evolution of the system and the increase of temperature may vary between a factor of 1.1 and 1.5 .

It is worthwhile mentioning that temperature and density enhancments could also be produced by adiabatic compression and vertical bi-directional flows can be formed via pressure increase when there are horizontal converging flows. This could be another mechanism (alternative to magnetic reconnection) responsible for the formation of small scale brightenings at various atmospheric heights (e.g. EBs). However, to show that this is not true in all cases in our numerical experiment, we calculate the time evolution (at three different times) of entropy across three possible sites of EBs (bottom panel in Fig. 8). Adiabatic compression is an isentropic process and, thus, entropy should not increase at the locations of density and temperature enhancment. However, entropy shows a pronounced increase at these locations while it remains aprroximately invariant in between them. Current density, temperature and plasma density have also local maxima at the same sites (see middle panel in Fig. 8). We should mention that we have also calculated the parallel electric field and we found that it has a maximum at the EBs. Although, we use a small explicit resistivity, the parallel electric field in narrow layers (the current sheets) can reach values that are a few hundred times larger than the average value of the background plasma. This means that resistive effects, such as magnetic reconnection, are very important at the locations of EBs.

Figure 9 shows the time variation of various energies (top panel) and the current density (bottom panel) around the site of a possible EB (located at $y \approx 65, x \approx 53$ and starting around $t=$ $80)$. The magnetic, thermal and kinetic energies are defined by

$$
\begin{aligned}
& E_{\text {magn }}=\iiint \frac{B^{2}}{8 \pi} \mathrm{d} x \mathrm{~d} y \mathrm{~d} z \\
& E_{\text {th }}=\iiint \frac{P}{\gamma-1} \mathrm{~d} x \mathrm{~d} y \mathrm{~d} z \\
& E_{\text {kin }}=\iiint \frac{1}{2} \rho\left[u x^{2}+u_{y}^{2}+u_{z}^{2}\right] \mathrm{d} x \mathrm{~d} y \mathrm{~d} z
\end{aligned}
$$

respectively. They are integrated in the region: $48<x<56$, $60<y<67$ and $3<z<9$. It should be noted that for the
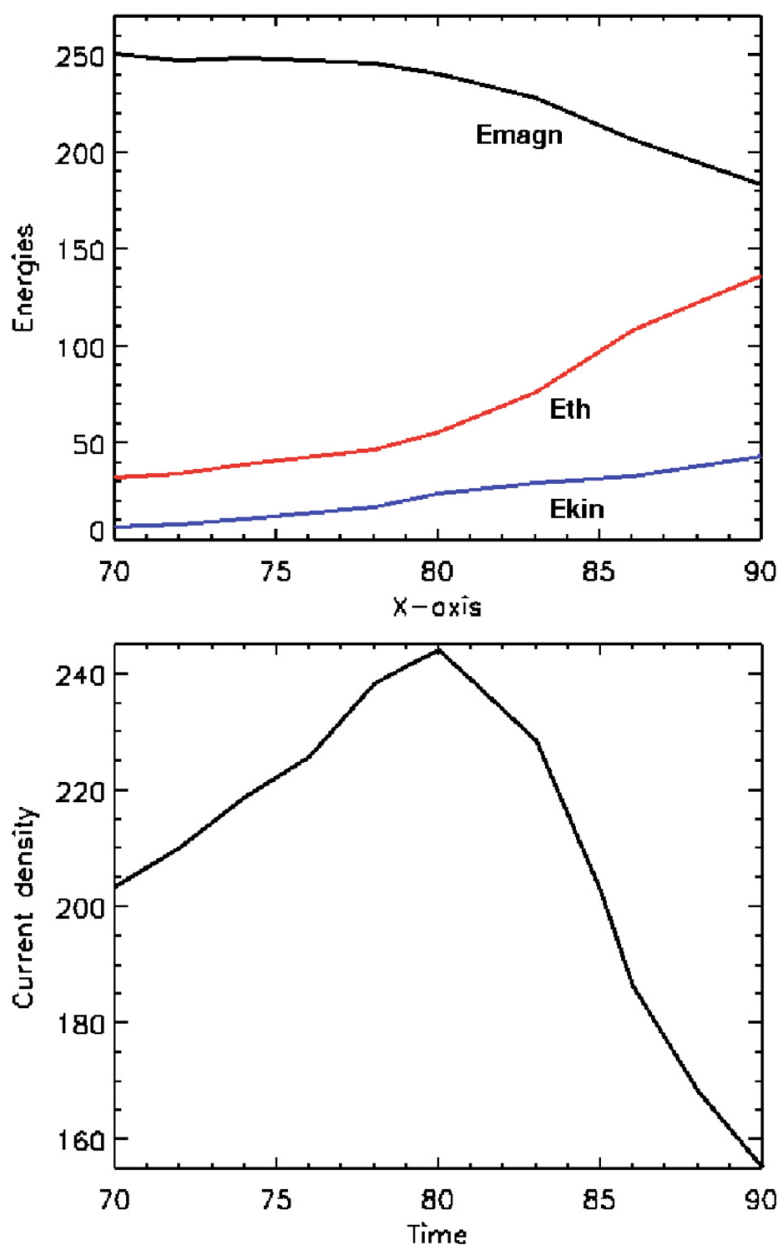

Fig. 9. Top: time evolution of various energies. Bottom: variation of current density along time.

thermal energy we calculate $\Delta E_{\mathrm{th}}=E_{\mathrm{th}}(t)-E_{\mathrm{th}}(0)$. For the current density, we calculate the maximum value of $|\boldsymbol{J}|$ within the integrated volume. For $70 \leq t \leq 80$ the maximum current builds up but the energies do not show any significant variation. Dissipation of the current starts at around $t=80$. For $t>80$, maximum current density drops and magnetic energy decreases. At the same time, both the thermal and kinetic energies increase. These changes in energy strongly indicate that magnetic reconnection is occuring. The decrease of magnetic energy during the period $80<t \leq 90$ agrees with the entropy increase around the same location of an EB, during the same time interval (see bottom panel, Fig. 8).

This result, together with the sudden change of connectivity of the fieldlines and the existense of bidirectional flows, strongly suggests that magnetic reconnection is the most plausible driving mechanism for these EBs that are formed in our experiment.

Based on the physical extent (along the $x$ - and $y$-directions) to which the temperature and density show a significant relative increase, one can estimate an average size for the aforementioned bright structures. From Fig. 7 this rough estimate gives a typical width ( $x$-size) between 4 and $5(\approx 720-900 \mathrm{~km})$ and a typical length between 4 and $6(\approx 720-1080 \mathrm{~km})$. The shape of these structures is mainly determined by the geometry of the magnetic fields that come into contact and produce them via reconnection. At sites where segments of three or more rising loops interact (e.g., $x=30, y=48$ in Fig. 7), they look like bright sources with a roundish shape. This is the most 
common shape that they adopt during the evolution of the system. However, there are other places where the interfaces between interacting magnetic fields are more stretched (e.g., $67<$ $x<78,50<x<68$ ) with an elongated shape, like bright lanes. The small-scale brightenings have an average life-time of $\Delta t=20$ (i.e. 6-7 min). In few cases, these features (e.g., at $x=20, y=30$ ) may exist for $\Delta t=34$ (i.e. $\approx 11 \mathrm{~min}$ ).

\subsection{Comparison with observations: Ellerman bombs}

The above characteristics of the small-scale bright features compare well with quantitative measurements of EBs. Kurokawa et al. (1982) found that EBs have a typical length of $\approx 1$ arcsec and are associated with photospheric downflows. Matsumoto et al. (2008) reported downflows at the photosphere $\left(\approx 0.2 \mathrm{~km} \mathrm{~s}^{-1}\right)$ and upflows in the chromosphere $\left(\approx 1-3 \mathrm{~km} \mathrm{~s}^{-1}\right)$. Dara et al. (1997) also reported strong upflows in the chromosphere. Georgoulis et al. (2002) investigated the statistical properties of EBs in an emerging flux region and found that EBs were correlated with downflows $\left(\approx 0.5 \mathrm{~km} \mathrm{~s}^{-1}\right)$ at the photosphere. Also, non-LTE analysis of spectral profiles of EBs showed strong downflows $\left(\approx 10 \mathrm{~km} \mathrm{~s}^{-1}\right.$ ) between the upper photosphere and the low chromosphere (Socas-Navarro et al. 2006). An interesting result was reported by Matsumoto et al. (2008) who performed spectroscopic observations of EBs in an active region in order to investigate the height dependence of gas flows in EBs. They found that bidirectional flows may start between the lower photosphere and the lower chromosphere. The above results are in agreement with our simulations, where bidirectional flows are produced (see Figs. 6 and 8) at various heights between photosphere and chromosphere.

Rust et al. (1977) found that some EBs are related to $\mathrm{H} \alpha$ surges, which are commonly thought to be the result of magnetic reconnection at the low chromosphere. Kurokawa et al. (1982) and Kitai (1983) also detected strong upflows $\left(\approx 8 \mathrm{~km} \mathrm{~s}^{-1}\right)$, which may account for small surges, at the location of Ebs. These observations are also consistent with the highvelocity upflows produced in our simulations, above the locations of EBs during the evolution of the system. At the region of EBs, Socas Navvaro (2005a) found strong currents, which indicates that EBs may be associated with reconnection. Figure 7 shows that regions with enhanced current density are favourable sites for the appearance of EBs in our numerical experiments.

Kitai (1983) found that a density enhancement (by a factor of 5$)$ and a hot temperature gradient $(\approx 1500 \mathrm{~K})$ may account for the line broadening in EBs. Georgoulis et al. (2002) and Pariat et al. (2004) also found that the temperature increases by $\approx 2000 \mathrm{~K}$ at the sites of EBs. In our simulations, the density enhancement is close to the value reported by Kitai (1983). The increase of temperature is larger, possibly due to the fact that the energy equation in our numerical experiments does not include all the thermodynamical terms (such as thermo-conduction) and the equation of state is for an ideal gas. Thermal and nonthermal semi-empirical atmospheric models for EBs have been presented by Fang et al. (2006) and Chen et al. (2001). They found that the temperature enhancement in the lower chromosphere is about $600-1300 \mathrm{~K}$. The increase of temperature was reduced by $100-300 \mathrm{~K}$ when the nonthermal effects were included in the model.

Finally, many observations have shown that the typical lifetime of EBs is 10-20 min (Kurokawa et al. 1982; Nindos \& Zirin 1998; Qiu et al. 2000). Pariat studied the UV time profiles of EBs and found that the distribution of lifetimes for individual impulses (associated with the EBs) peaks between 3

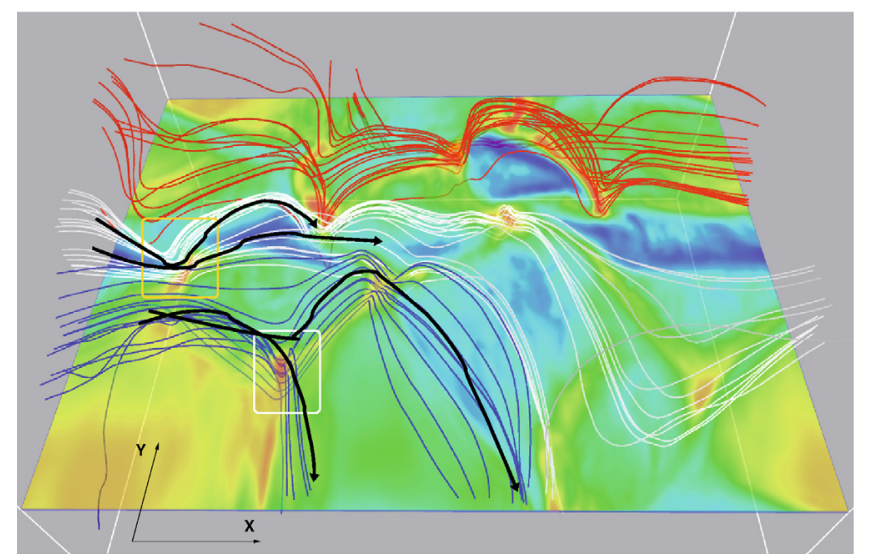

Fig. 10. 3D topology of magnetic fieldlines as the undulating magnetic flux system emerges through the atmosphere $(t=90)$. The temperature distribution at the low chromosphere $(z=6)$ is shown by the horizontal slice. The latter extends across the whole numerical domain, in the $x$ and $y$-directions.

and $4 \mathrm{~min}$. In our simulations, the lifetime of the EBs is between 6 and $11 \mathrm{~min}$.

\subsection{Topology around Ellerman bombs}

Figure 10 shows a number of fieldlines that have been traced from the EBs, at the low chromosphere at $t=90$. The horizontal slice shows the distribution of temperature with the same scaling as that in Fig. 7. The 3D visualization of the topology of the field reveals that the majority of the EBs are found in locations where the magnetic fieldlines have a V-shape. Note, that a substantial number of fieldlines possess more than one V-like dips along their length and, thus, many EBs appear to be inter-connected with the same fieldlines. As we have shown in Sect. 3.2, effective reconnection, which occurs at the locations of EBs, is essential for the emergence of the magnetic flux system into the higher atmosphere. The general topology of the magnetic field in association with the location of the EBs is reminiscent of the model proposed by Pariat et al. (2004) who reported that the dips of undulatory flux tubes are cospatial with EBs.

Another interesting topological feature in Fig. 10 is the dramatic change of connectivity of fieldlines around some of the sites of EBs. For example, notice the sudden change in the connectivity of the blue and white fieldlines that they pass through the EBs in the locations marked with rectangular boxes. The long overplotted (black) arrows show the direction of the full magnetic field vector along individual sets of fieldlines. It is likely that in such complex topologies, quasi-seperatrix layers (QSLs) are formed at the interfaces between neighbouring magnetic systems that interact during the emergence of the field. The concept of current sheet formation and reconnection in QSLs has been studied extensively in the past (Priest \& Démoulin 1995; Aulanier et al. 2005; Démoulin 2006). Therefore, it is expected that QSLs may occur in the low atmosphere and EBs are triggered somewhere along QSLs. EB triggering by magnetic reconnection on QSLs was also favoured by the observational data obtained by Georgoulis et al. (2002).

\section{Late evolution of the magnetic field}

In this section we study the late evolution $(t \geq 70)$ of the magnetic field at the photosphere. At the beginning, the magnetic 

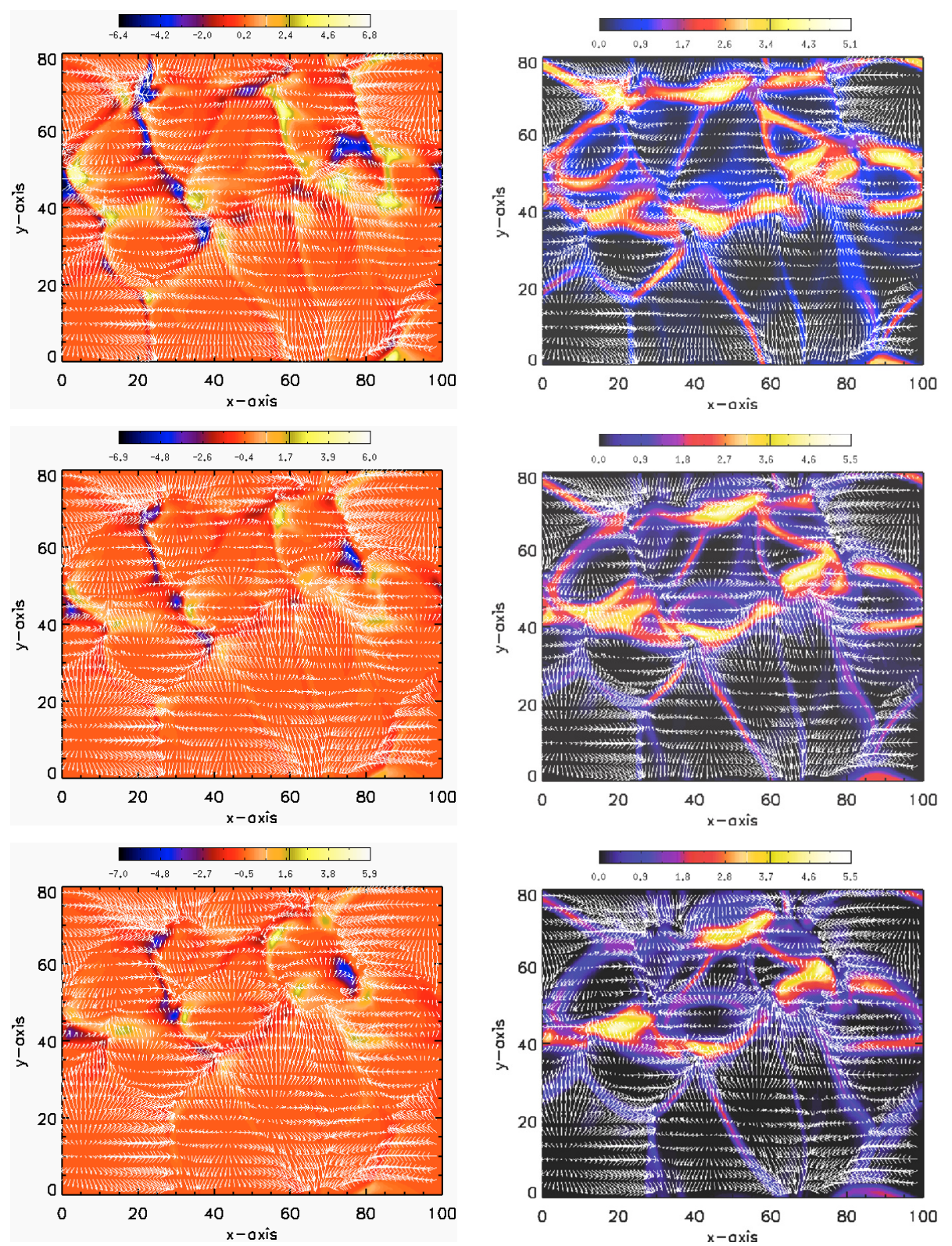

Fig. 11. Left: distribution of $B_{z}$ at the photosphere for $t=70, t=80$ and $t=90$ (from top to bottom). The projection of the horizontal velocity field vector on the slice is shown by the arrows. Right: distribution of $B_{\text {hor }}$ for the same height and times.

field is emerging at the photosphere in the form of horizontal field with an average field strength of about $600 \mathrm{G}$. The horizontal field is stronger than the vertical magnetic field. Until, $t \approx 55$, the horizontal field constitutes almost $90 \%$ of the total magnetic field strength, in most of the photospheric layer at $z=1$. The magnetic field continues to emerge and start to rise into the outer atmosphere, in the form of magnetic loops. As the emerging loops rise, the magnetic field is moving from the initial site of emergence towards the flanks of the emerging loops and, thus, it adopts a more pronounced vertical configuration. At $t=70$, the horizontal magnetic field is still more space-filling than the vertical magnetic field. However, the strongest field (e.g., field with values larger than $80 \%$ of the maximum value of $|\boldsymbol{B}|)$ is nearly all vertical. After $t=70$, the average values of the vertical and the horizontal magnetic field at the photosphere do not change dramatically.

\subsection{Intensification of the magnetic field}

Although the estimate of the average magnetic field does not go under significant changes, there are still regions at the photosphere where, after $t=70, B_{\text {hor }}$ and $B_{z}$ increase locally. The topology of the flows plays a role in the local increase of the field. In the following, we will present examples of flux concentrations, that undergo intensification during the late evolution of the system.

Figure 11 (left column) shows the time evolution of $B_{z}$ at the photosphere. The horizontal velocity field is overplotted (arrows) on the photospheric plane. The magnetic field is emerging at various places and then it expands in the atmosphere. The expansion of the field drives horizontal outflows away from each emerging region. The overal topology of the velocity field shows that the horizontal outflows are converging at the boundaries between neighbouring emerging regions. Also, due to the $\Omega$-like shape of the emerging loops, there is drainage of mass (and, thus, downflows) along the edges of the loops.

The site between $x=75-80$ and $y=55-60$ is located between three emerging loops. The dominant polarity of the magnetic field in this region is negative. Figure 11 (top panel) shows the enhancement of $B_{z}$ : at $t=60$ the negative flux concentration has field strength around $3(\approx 900$ Gauss $)$ and at $t=80(6-7 \mathrm{~min}$ later) the magnetic field has strength as high as $7(\approx 2 \mathrm{kG})$. The 

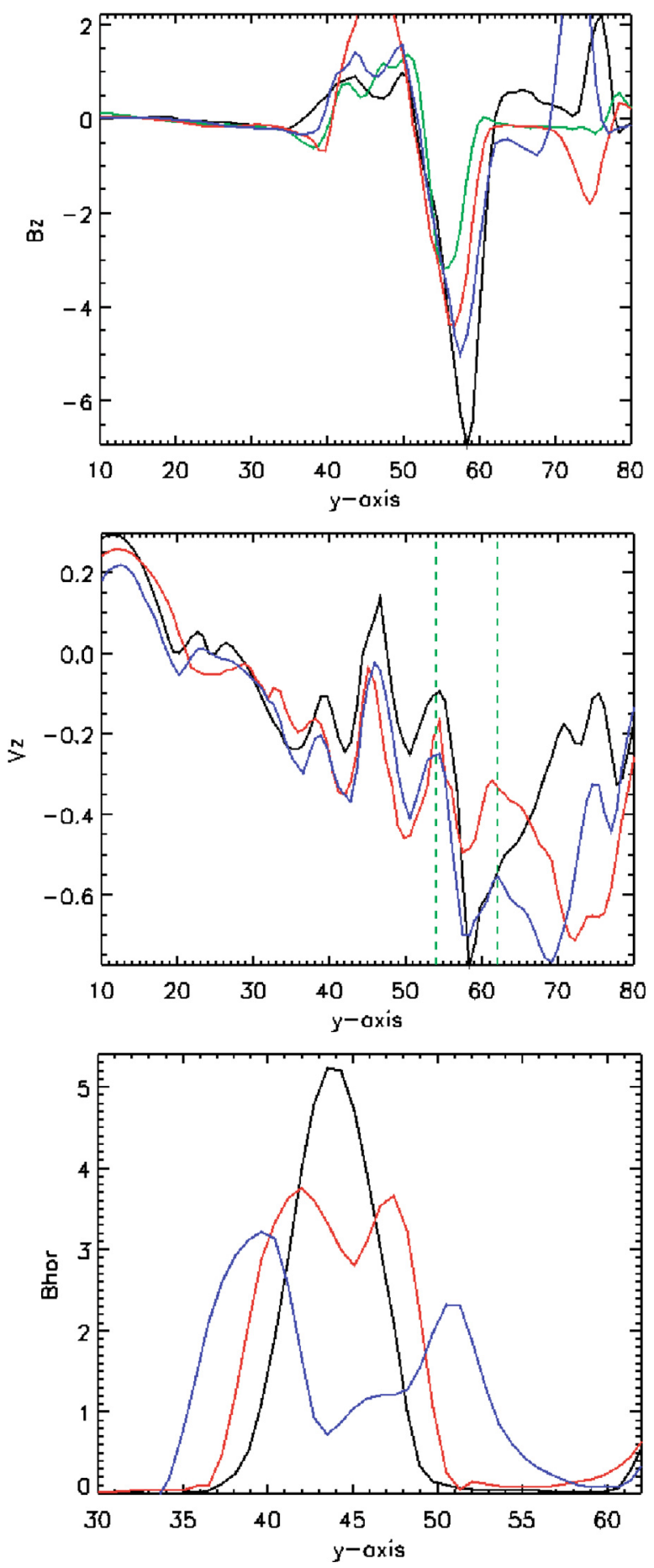

Fig. 12. Top: distribution of $B_{z}$ along the $y$-direction at $x=77$ and $z=1$. Times are: $t=60$ (green), $t=70$ (red), $t=75$ (blue) and $t=80$ (black). Middle: distribution of $v_{z}$ in the same region for $t=70$ (red), $t=75$ (blue) and $t=80$. Bottom: intensification of $B_{\text {hor }}$ at photospheric heights $(z=1)$. Times are: $t=70$ (blue), $t=80$ (red) and $t=90$ (black).

middle panel in Fig. 12 shows the distribution of $V_{z}$ in this region. The magnetic field is enhanced where strong downflows exist. The speed of the donflows increases from 0.5 to $\approx 0.8$ (4.5 to $7 \mathrm{~km} \mathrm{~s}^{-1}$ ) between $t=70$ and $t=80$. The acceleration of the downflows is due to the dense plasma that is drained through the footpoints of the loops and the superadiabaticity of the atmosphere. Also, the converging flows, which are caused due to the expansion of nearby emerging fields are $\approx 2 \mathrm{~km} \mathrm{~s}^{-1}$ and $\approx 5 \mathrm{~km} \mathrm{~s}^{-1}$ along the $x$ - and $y$-direction respectively.

Due to the strong downflows and the effective drainage of mass, the plasma becomes partially evacuated in the upper layers (e.g, the density is less at $z=1$ than at $z=-1$ ). Also, the density in this region is less by a factor of $\approx 0.8$ than the average density of the background atmosphere. The relative decrease in the gas pressure is a factor of $\approx 0.7$. Thus, the converging outflows from the expanding loops are able to compress the fluid and strengthen the magnetic field. Intensification stops when pressure balance is achieved between the magnetic flux concentration and the external atmosphere.

The horizontal magnetic field can be intensified by a similar mechanism. Figure 11 (right column) shows that the field is horizontal mainly at the boundaries between emerging magnetic loops. The cross section of two loops that emerge to the photosphere at different locations are those close to $x=15, y=55$ and $x=22, y=30$ respectively (top-right panel in Fig. 11). As time goes on, the magnetic field expands and the edges of the rising loops come into contact (bottom-right panel). This is when the horizontal segments of the magnetic field, which belong to the interacting emerging loops, are folded constructively since they have almost parallel orientation. Again, the fluid at the interface is compressed by the converging motions and the strength of the field increases accordingly. The bottom panel in Fig. 12 shows the distribution of the horizontal magnetic field along the $y$-direction at $x=18.7$ for three different times. The two local maxima correspond to the horizontal segments of the two emerging loops. At this stage of the evolution, $B_{\text {hor }}$ is close to 3.2 (between 900-1000 G). Eventually, they approach to each other and collide, increasing the magnitude of the horizontal field to $\approx 5.3$ (1590 G).

\subsection{New emergence of flux}

A possible consequence of the convectively unstable layer below the photosphere is the emergence of new magnetic flux, driven by convective upflows. An episode of emergence of new magnetic flux is shown in Fig. 11. At $t=70$ the uppermost layers of the emerging field have reached the photosphere $(x=45, y=$ 42). As the magnetic field continues to rise, it expands laterally and modifies the preexisting flow pattern at the photosphere. The upward rising motion appears as an upflow cell that eventually induces diverging (down)flows at the periphery of the emerging field. The area of the emerging field, when it rises initially at the photosphere, is $\approx 15 H_{\mathrm{ph}} \times 15 H_{\mathrm{ph}}$.

The emergence of the magnetic field into the higher atmosphere is shown in Fig. 13 (top panel). We plot the distribution of the total magnetic field strength along height at the center of the cross section of the emerging field for $t=70,80$ and 90. The field strength, when it firstly crosses the photospheric layer, can be as high as $450 \mathrm{G}$. Eventually, as it expands, the field strength drops (under the condition of magnetic flux conservation) to $320 \mathrm{G}$ at $t=90$. By that time, a considerable amount of emerging field has been advanced into the higher levels of the atmosphere.

The rise of the new emerging field into the corona does not proceed uninhibited. The earlier dynamical rise and expansion of neighbouring magnetic loops has formed a pre-existing ambient field in the corona. The interaction between the new emerging field and the pre-existing magnetized atmosphere produces rotational discontinuities, which might lead to the development of layers with enhanced current density. The appearance of bright 

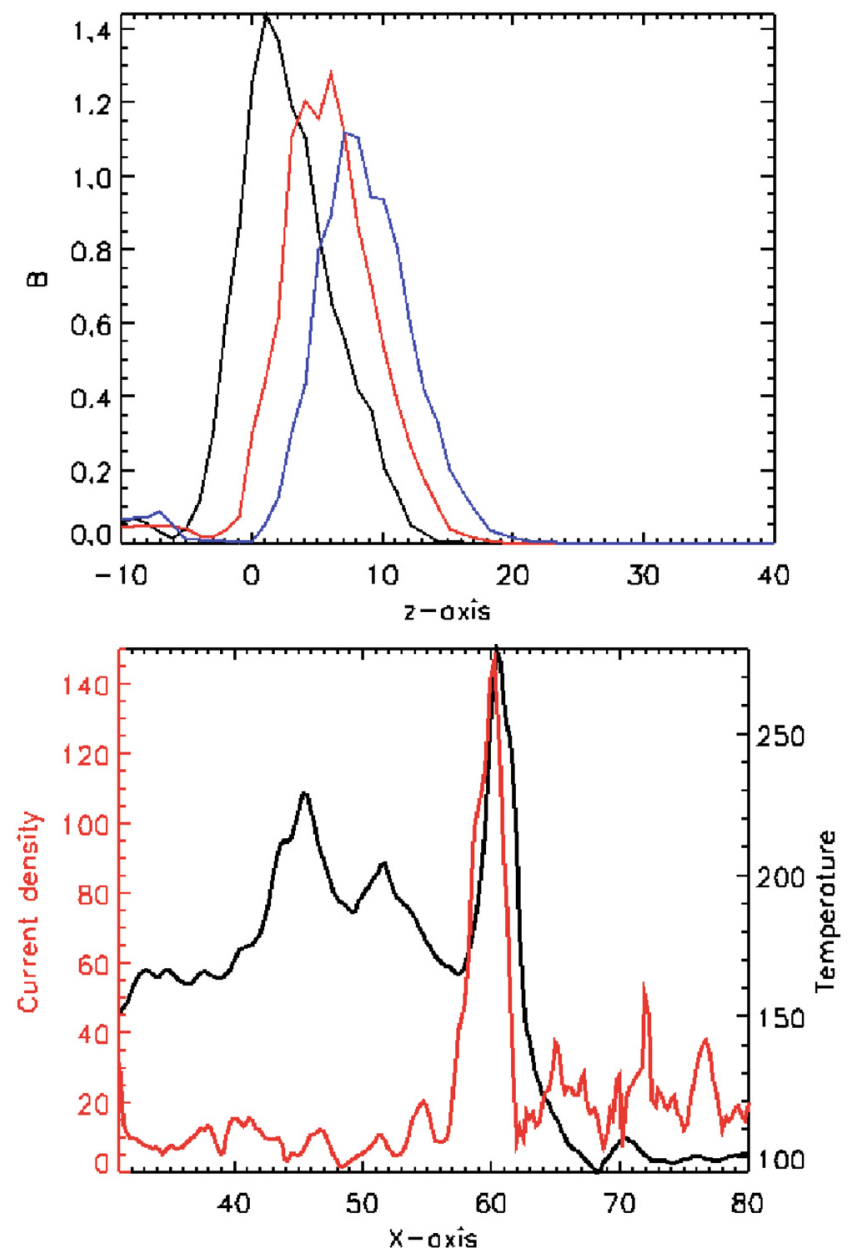

Fig. 13. Top: the distribution of the total magnetic field strength along height as it is calculated at the central part of the new flux emerging region. The distribution is shown for $t=70$ (black), $t=80$ (red) and $t=$ 90 (blue). Bottom: distribution of temperature (black line) and current density (red) along the $x$-axis, at $z=20$ and $t=90$.

structures in the corona could be the result of the local enhancement in the temperature of these layers.

The bottom panel in Fig. 13 shows the increase of temperature in the corona when a current layer is formed at the interface between the emerging flux and ambient field. The ambient field is formed by the expansion of magnetized plasma that belongs to a neighbouring flux system. The local maximum of the current density (red line) at $z \approx 60$ correlates well with the enhancement of the temperature, which could be a factor of 2.8 higher than the initial coronal temperature $(\approx 1 \mathrm{MK})$.

In our numerical experiments, rotational discontinuities in the higher atmosphere are formed not only when new magnetic flux interacts with ambient field but also due to the very dynamical interaction between neighbouring emerging loops. The fieldlines of the expanding loops are free to move in three dimensional space and, thus, it is not unlikely that sets of fieldlines with opposite orientations press against each other and form strong current sheets. An example of this type of interaction between emerging fields is given in Fig. 4. The region between the loops L3 and L4 (marked with an arrow, at $t=70$ and $t=90$ ) is where current density increases, followed by an enhancement of the temperature in the corona. The $3 \mathrm{D}$ interaction between an emerging twisted flux tube (or flux sheet) and a pre-existing field in the corona has been studied in more detail by Archontis \& Hood (2008), Galsgaard et al. (2005) and Isobe et al. (2005).

\section{Summary and discussion}

Through our numerical experiments we have investigate the emergence of a magnetic flux system with undulations in three dimensions. Magnetic fields with a sea-serpent configuration appear in various places in the solar surface e.g., in the central part of an emerging active region. We show that the emergence into the corona occurs through reconnection events at successive atmospheric heights, from the photosphere up to the outer solar atmosphere. This resistive emergence process leads to the formation of large-scale magnetic flux systems, with sizes of a few tens of $\mathrm{Mm}$, which rise into the corona. The magnetic field is free to emerge and expand, since the dense plasma, which accumulates at the dips between undulations, is off-loaded in the low atmosphere by reconnection.

By-products of the efficient reconnection process are smallscale brightenings, which appear at various atmospheric heights. In the upper photosphere/chromosphere, these events can account for Ellerman bombs. Our numerical results show a good comparison with some of the major characteristics of EBs as recorded by observations. However, for a direct comparison with observational data, extra thermodynamical aspects need to be included in the numerical models, since the heat loss/gain due to radiative cooling and heat conduction are important. Also, the inclusion of partial ionization could play a role in the formation of current sheets and the temperature enhancement at the sites of EBs. In the upper atmosphere, brightenings appear due to the dynamical interaction between newly emerging flux and preexisting coronal field or between emerging fieldlines that come into contact and form strong current layers.

One difference with the corresponding 2D numerical study (Isobe et al. 2007) is the development of interchange modes for the magnetic field and the excitation of non-linear, dynamic coupling between emerging fields. This leads to the formation of a complex network of rising loops that emerge into the atmosphere over a range of temporal and spatial scales. Moreover, a series of interesting phenomena are produced such as: the formation of many structures with enhanced current density and a series of bright features throughout the atmosphere, the existence of bi-directional flows that in few cases may reach high velocities, flux cancellation at the low atmosphere and so on.

The appearance of magnetic field undulations in our experiments is due to the Parker instability, which is triggered by the initial velocity perturbation on the uniform magnetic flux sheet. The convectively unstable layer supports the formation of the undulations, which eventually grow forming magnetic loop-like structures that emerge through the photosphere. However, in our experiments the sub-photospheric layer is not a realistic convective envelope. Other 3D MHD radiative simulations, that include a convection zone (Cheung et al. 2008), identify convective dynamics as the main reason for the formation of serpentine-like fieldlines at the photosphere. More observations and numerical studies are required for a detailed study of this issue. On the other hand, we assertain that the further rise of the magnetic field into the higher atmosphere is independent of the cause for the appearance of undulations at the photosphere.

The general evolution of the magnetic field in our model is similar to those in the resistive emergence model proposed by Pariat et al. (2004). Although there is a good comparison between our numerical results, the theoretical model by Pariat et al. (2004) and relevant observations, more experiments are required 
to study the effect of varying the parameters used in our simulations such as, the field strength, thickness and location of the flux sheet, the superadiabaticity and the inclusion of extra heating/cooling sources in the energy equation.

Acknowledgements. We gratefully acknoweledge the financial support of STFC and the European Commission through the SOLAIRE Network (MTRN-CT2006-035484). The simulations were performed on the STFC and SRIF funded UKMHD cluster, at the University of St Andrews.

\section{References}

Archontis, V., \& Hood, A. W. 2008, ApJ, 674, L113

Archontis, V., Moreno-Insertis, F., Galsgaard, K., Hood, A., \& O'Shea, E. 2004, A\&A, 426, 1047

Aulanier, G., Pariat, E., \& Démoulin, P. 2005, A\&A, 444, 961

Bernasconi, P. N., Rust, D. M., Georgoulis, M. K., \& Labonte, B. J. 2002, Sol. Phys., 209, 119

Chen, P.-F., Fang, C., \& Ding, M.-D. D. 2001, Chinese J. Astron. Astrophys., 1, 176

Cheung, M. C. M., Schüssler, M., Tarbell, T. D., \& Title, A. M. 2008, ApJ, 687, 1373

Dara, H. C., Alissandrakis, C. E., Zachariadis, T. G., \& Georgakilas, A. A. 1997, A\&A, 322, 653

Démoulin, P. 2006, Adv. Space Res., 37, 1269

Ding, M. D., Henoux, J.-C., \& Fang, C. 1998, A\&A, 332, 761

Ellerman, F. 1917, ApJ, 46, 298

Fang, C., Tang, Y. H., Xu, Z., Ding, M. D., \& Chen, P. F. 2006, ApJ, 643, 1325

Galsgaard, K., Moreno-Insertis, F., Archontis, V., \& Hood, A. 2005, ApJ, 618, L153
Georgoulis, M. K., Rust, D. M., Bernasconi, P. N., \& Schmieder, B. 2002, ApJ, 575,506

Harvey, K., \& Harvey, J. 1973, Sol. Phys., 28, 61

Isobe, H., Miyagoshi, T., Shibata, K., \& Yokoyama, T. 2005, Nature, 434, 478

Isobe, H., Miyagoshi, T., Shibata, K., \& Yokoyama, T. 2006, PASJ, 58, 423

Isobe, H., Tripathi, D., \& Archontis, V. 2007, ApJ, 657, L53

Kitai, R. 1983, Sol. Phys., 87, 135

Kurokawa, H., Kawaguchi, I., Funakoshi, Y., \& Nakai, Y. 1982, Sol. Phys., 79, 77

Mackay, D. H., Longbottom, A. W., \& Priest, E. R. 1999, Sol. Phys., 185, 87

Mandrini, C. H., Démoulin, P., Schmieder, B., Deng, Y. Y., \& Rudawy, P. 2002, A\&A, 391, 317

Matsumoto, T., Kitai, R., Shibata, K., et al. 2008, PASJ, 60, 95

Murray, M. J., Hood, A. W., Moreno-Insertis, F., Galsgaard, K., \& Archontis, V. 2006, A\&A, 460, 909

Nindos, A., \& Zirin, H. 1998, Sol. Phys., 182, 381

Nozawa, S., Shibata, K., Matsumoto, R., et al. 1992, ApJS, 78, 267

Pariat, E., Aulanier, G., Schmieder, B., et al. 2004, ApJ, 614, 1099

Pariat, E., Schmieder, B., Berlicki, A., et al. 2007, A\&A, 473, 279

Parker, E. N. 1966, ApJ, 145, 811

Priest, E. R., \& Démoulin, P. 1995, J. Geophys. Res., 100, 23443

Qiu, J., Ding, M. D., Wang, H., Denker, C., \& Goode, P. R. 2000, ApJ, 544, L157

Rust, D. M., Webb, D. F., \& MacCombie, W. 1977, Sol. Phys., 54, 53

Seehafer, N. 1986, Sol. Phys., 105, 223

Shibata, K., Tajima, T., Matsumoto, R., et al. 1989, ApJ, 338, 471

Socas-Navarro, H., Martínez Pillet, V., Elmore, D., et al. 2006, Sol. Phys., 235, 75

Spruit, H. C., Title, A. M., \& van Ballegooijen, A. A. 1987, Sol. Phys., 110, 115

Titov, V. S., \& Démoulin, P. 1999, A\&A, 351, 707

Watanabe, H., Kitai, R., Okamoto, K., et al. 2008, ApJ, 684, 736

Zwaan, C. 1985, Sol. Phys., 100, 397 